\title{
CME Genetic medicine (113042) self-assessment questionnaire
}

\author{
Edited by Julian Barwell and Tahseen A Chowdhury
}

SAQs and answers are ONLINE for RCP fellows and collegiate members

SAQs and answers are ONLINE for RCP fellows and collegiate members

\section{Format}

Candidates are asked to choose the best answer from the five possible answers. This best of five format is used in many medical examinations; however, the questions are not intended to be representative of those used in the MRCP(UK) Part 1 or Part 2 Written Examinations.

\section{The answering process}

1 Go to www.rcplondon.ac.uk/SAQ

2 Log on using your usual RCP username and password

3 Select the relevant CME question paper

4 Answer all 10 questions by selecting the best answer from the options provided

5 Once you have answered all the questions, click on Submit

\section{Registering your external CPD credits}

Carrying out this activity allows you to claim two external CPD credits. These will be automatically transferred to your CPD diary, where you can review the activity and claim your points.

1. Whole genome sequencing (WGS) has the potential to detect different types of genetic variation. Please indicate which one of the genetic variants would remain undetected via this test.
(a) Copy number variants
(b) Epigenetic variants
(c) Mitochondrial sequence variants
(d) Non-coding variants
(e) Single nucleotide variants

2. A 35-year-old woman diagnosed with high-grade breast cancer at 33 years is attending the oncology clinic for counselling and consideration of genetic testing. Her paternal aunt was diagnosed with breast cancer at 39 years and her paternal uncle died at 55 years due to a brain tumour. She has a 6 year old daughter recently diagnosed with a soft tissue sarcoma.
Which of the following genes would you sequence as a priority?
(a) BRCA1
(b) BRCA2
(c) TP53
(d) PTEN
(e) PALB2

3. A 23-year-old male patient presents with a 1-year history of developing several smooth firm skin coloured papules around his nails and associated with a longitudinal groove on affected nails. He had a history of hypopigmented areas on his torso and also a history of small pink red papules over his cheeks and nose since childhood. He also had a background history of epilepsy and has a mild learning disability. Which is the most likely unifying diagnosis?
(a) Birt-Hogg-Dube
(b) Familial leiomyomas
(c) Cowden syndrome
(d) Tuberous sclerosis
(e) Muir-Torre syndrome

4. A 31-year-old woman was seen in the genetics clinic shortly after delivery of her baby. Antenatally there was cystic hygroma, and amniocentesis had identified a normal male karyotype. Her baby was found to have lymphoedema of the feet at birth. The woman had a history of learning difficulties and had pulmonary stenosis under Cardiology review. What is the most likely diagnosis in the family?
(a) Emberger syndrome
(b) Hennekam syndrome
(c) Lymphoedema distichiasis syndrome
(d) Noonan syndrome
(e) Turner syndrome

5. A 36-year-old woman with colorectal cancer has consented to undergo whole genome sequencing of her tumour DNA and germline DNA. At present, she has only consented to receive information relevant to her current diagnosis. Which of the following would be most appropriate to seek from her germline DNA for variant interpretation with a view to reporting back to the patient?
(a) A missense variant in the gene BRCA1
(b) A nonsense mutation in MSH2, a DNA mis-match repair gene 
(c) A single nonsense sequence variant in the gene MUTYH which in biallelic form causes multiple colorectal adenomas

(d) A single nucleotide deletion in the gene LDLR, which causes familial hypercholesterolaemia

(e) The presence of the BRAF V600E mutation

6. A 22-year-old man is seen in your clinic. From birth he had hypertrophy of his left leg with an associated epidermal naevus, lipomas and scoliosis (CLOVES). You wish to arrange genetic testing to confirm the diagnosis. What is the most appropriate genetic test to arrange?

(a) A blood test for a panel of genes associated with lymphoedema

(b) A blood test for chromosome analysis (karyotype)

(c) A saliva sample for a panel of genes associated with lymphoedema

(d) A skin biopsy from the right leg sent for testing of a panel of genes in the PI3K/AKT/mTOR pathway

(e) A skin biopsy from the left leg sent for testing of a panel of genes in the PI3K/AKT/mTOR pathway

7. A 36-year-old male patient presents with a several years history of developing multiple yellow white papules on his neck, upper trunk and mid face. He has a family history of renal cell carcinoma and a personal history of a previous pneumothorax. What is the most likely underlying unifying diagnosis?
(a) Familial leiomyomas
(b) Cowden syndrome
(c) Birt-Hogg-Dube
(d) Tuberous sclerosis
(e) Muir-Torre syndrome

8. A 37-year-old women with personal and family history of breast cancer underwent gene panel testing for suspected hereditary breast cancer. Her test identified a missense mutation in PALB2 gene. Which of the following information would be useful for interpretation of her test result?

(a) In silico prediction analysis for the effect of the amino acid change to the protein product

(b) Genetic testing in other affected relatives with breast cancer for the detected PALB2 mutation

(c) Conservation data for the affected amino acid in other species (d) Assessment of the PALB2 mutation frequency in other affected and non-affected cohorts

(e) All of the above.

9. Which of the following statements is true regarding panel testing in hereditary breast and ovarian cancer?

(a) Panel tests typically identify genetic causes in $>30 \%$ of BRCA1/2-negative families

(b) The frequency of variants of uncertain significance (VUS) in panel tests is lower than single gene tests

(c) There is no limit to the number of genes that can be put on a panel.

(d) The absence of a pathogenic variant excludes the presence of familial and/or other inherited susceptibility factors.

(e) Panel testing can be offered without genetic counselling on possible outcomes and implications to at-risk relatives.

10. A 36-year-old female patient was initially referred to the breast clinic following the development of a new breast lump. Following investigation in addition to bilateral fibrocystic disease she was found to have a suspicious breast lesion, which on biopsy was a malignant tumour. She described a several years' history of the development of thickened papules around her nose and ears and also scaly red lesions on her bilateral palms and she was referred for dermatological assessment. Which is the most likely unifying diagnosis?
(a) Birt-Hogg-Dube
(b) Bazex syndrome
(c) Cowden syndrome
(d) CYLD cutaneous syndrome
(e) Muir-Torre syndrome

\section{CME Nuclear medicine SAQ}

Answers to the CME SAQ published in Clinical Medicine in October 2017

$\begin{array}{llllllllll}\text { Q1 } & \text { Q2 } & \text { Q3 } & \text { Q4 } & \text { Q5 } & \text { Q6 } & \text { Q7 } & \text { Q8 } & \text { Q9 } & \text { Q10 } \\ \text { (a) } & \text { (a) } & \text { (b) } & \text { (a) } & \text { (c) } & \text { (b) } & \text { (c) } & \text { (c) } & \text { (c) } & \text { (d) }\end{array}$

